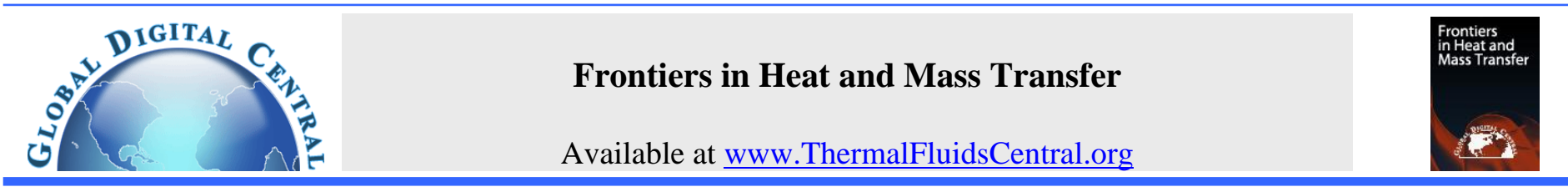

\title{
EXPERIMENTAL VALIDATION OF NATURAL CONVECTION IN A RECTANGLE USING SCHLIEREN IMAGING
}

\author{
Patrick C. Doherty, Heather E. Dillon ${ }^{\dagger}$, Justin Roberts \\ University of Portland, Portland, Oregon, 97203, USA
}

\begin{abstract}
The onset of turbulence in natural convection systems is difficult to predict using traditional computational techniques. The flow patterns that occur before and after the onset of turbulence may be better understood with the help of visual techniques like Schlieren imaging. Schlieren imaging allows visualization of the density gradients of a fluid using collimated light and refractive properties. In this experiment, a device was designed to test the behavior of airflow with non-isothermal boundary conditions within a rectangular cavity. Previous computational fluid modeling suggested a period doubling route to chaos in a cavity with a high aspect ratio and free convection flow driven by two non-isothermal walls. To visualize this system, two strip heaters were attached to two $56 \mathrm{~cm}$ long plates. The plates were set to an aspect ratio of 10 and sealed with gaskets and garolite. With a Z-type Schlieren device, the existence of two definitive cells was confirmed, and the oscillation point between the two cells was found above the cavity midpoint. At steady state, a thin-wire thermocouple was placed at the inflection point to measure fluctuations in temperature. Consistent oscillations in temperature were observed that indicated a steady interacting boundary layer, while non-periodic oscillations indicated the initial transition to turbulence. It was observed that there was a transition from a mostly steady interacting boundary layer at set-point $35^{\circ} \mathrm{C}$ to near-turbulence at $65^{\circ} \mathrm{C}$. At higher Rayleigh numbers the transition behavior predicted in the computational fluid model was confirmed, although the size of the convection cells was not uniform.

Keywords: Schlieren, convection, transition, visualization
\end{abstract}

\section{INTRODUCTION}

Although many theoretical approaches have been used to predict natural convection flow patterns of air, few have been proven through nondisturbing visual techniques. One such technique is Schlieren imaging. Schlieren imaging is a fluid visualization method that works by collimating light through a test region. Light is diffracted where the density (or temperature) changes in this test region. This effect produces various shades of light when viewed from around the focal point, allowing one to visualize change in the density of a fluid (Kaessinger et al., 2014).

Schlieren imaging as we know it today emerged from experiments done by J. B. Leon Foucault in 1859. Foucault discovered a knife-edge test for measuring the conic shapes of optic telescope mirrors to submicrometer accuracy (Settles, 2001). It was the first time an explicit cutoff, external to the human eye, was used to filter for Schlieren imaging (Settles, 2001). German physicists expanded on Foucault's test and discovered that it could be used to filter light, thus allowing one to visualize shadowy "streaks," or Schlieren, caused by light diffracting through areas of varying density (Settles, 2001). In the 20th Century, it was used to study the flow of air and shock-waves from planes in aeronautical engineering, but has been used much less as computer modeling software became widespread (Kaessinger et al., 2014). Experiments like the one discussed in this paper show Schlieren visualization can be useful for modern day studies to confirm computational fluid models and improve knowledge of complex applications. The primary advantage of Schlieren imaging is the ability to photograph, measure, and view the flow without disturbing the flow with a probe.

\footnotetext{
${ }^{\dagger}$ Corresponding author. Email: dillon@up.edu
}

In recent times, fluid fl ow be havior is of ten predicted an d visualized using computational fluid dynamics (CFD). The Schlieren imaging technique may be used to validate (or refute) these models. In this study, research was conducted to better characterize the convective flow in a non-isothermally heated rectangular cavity with an aspect ratio of 10 . This cavity was made of heated vertical walls and adiabatic horizontal boundary conditions. The temperature gradient studied was ideally linear across the two sides of the walls. CFD modeling in COMSOL by Dillon et al. (Dillon et al., 2013, 2009) predicted that two air cells would form in the air cavity region with an oscillating zone in the middle. Measuring the temperature at the point of oscillation and visualizing the airflow with a Schlieren device was used to determine the validity of the CFD model. Thus, the purpose of this experiment was to verify the existence of two oscillating cells, determine the location of the oscillation point between the cells, and find the onset of turbulence using a Schlieren imaging device.

\section{BACKGROUND}

There are three main types of Schlieren imaging techniques: the classical optical system, the focusing Schlieren optical system, and the backgroundoriented technique. The classical optical system is the type used in this experiment. This type always features one light source and usually a knife-edge at the cutoff plane. One variation of this type is the color Schlieren technique, which places a multi-color filter at the cutoff plane to make density variations appear more colorful and thus more identifiable (Settles, 1985). The focusing Schlieren optical system usually incorpo- 
Table 1 Summary of Schlieren imaging experiments used to visualize natural convection. ${ }^{*}$ Wollaston prism Schlieren interferometer with hologram projection.

\begin{tabular}{lclll}
\hline Author & Year & Schlieren Type & Experiment Type \\
\hline $\begin{array}{l}\text { Lewis et al. } \\
\text { (Lewis } \text { et al., 1969) }\end{array}$ & 1969 & & Convection around human head \\
$\begin{array}{l}\text { Aung, Fletcher, Sernas } \\
\text { (Aung } \text { et al., 1972) }\end{array}$ & 1972 & Linear lens* & Vertical parallel plate channels with asymmetric heating \\
$\begin{array}{l}\text { Okhotsimskii and Hozawa } \\
\text { (Okhotsimskii and Hozawa, 1998) }\end{array}$ & 1998 & Linear lens & Natural convection in binary gas-liquid systems \\
$\begin{array}{l}\text { Kaessinger et al. } \\
\text { (Kaessinger } \text { et al., 2014) }\end{array}$ & 2014 & Z-type & Convection over heated cylinder \\
$\begin{array}{l}\text { Tanda et al. } \\
\text { (Tanda } \text { et al., 2014) }\end{array}$ & 2015 & Z-type & Water natural convection in a vertical ribbed channel \\
Present work & 2016 & Z-type & Convection in rectangular cavity, non-isothermal boundary conditions & $T, t$ \\
\hline
\end{tabular}

rates an illumination pattern with multiple edges, rather than collimated light and a single knife edge (Settles, 2001). This allows one to visualize solely the distortions of interest (Settles, 2001). The background-oriented technique relies on visualizing shifts in focused images with a set background. Differences between subsequent images can be recorded and analyzed using numerical computing software, as done by Venkatakrishnan and Meier (Venkatakrishnan and Meier, 2004). The background oriented Schlieren technique will be considered for a future study of the geometry discussed in this paper.

In addition, there are three main types of Schlieren setups: linear lens, V-type, and Z-type. Foucault accidentally discovered the first one, linear lens. As the name suggests, this type is lined up linearly and employs two lenses to collimate light through the test region. Kaessinger et al. (Kaessinger et al., 2014) considered this setup for a similar natural convection study, but encountered size and image quality constraints. They then decided on the Z-type setup used in this experiment. Rather than lenses, the Z-type setup uses two parabolic mirrors. Though it is more space intensive, the Z-type has a greater potential to produce larger, more detailed images with larger subjects (Kaessinger et al., 2014). The V-type setup is similar to the Z-type, but with only one mirror.

Schlieren imaging has proven useful in several other documented free convection studies as summarized in Table 1. For instance, Lewis, Foster, and Mullan (Lewis et al., 1969) captured Schlieren images of the human head in standing and supine positions. This study was able to note marked differences in flow velocities and thermal boundary layer thicknesses between those two positions (Clark and Toy, 1975). The study by Kaessinger et al (Kaessinger et al., 2014) measured the boundary layer thickness of a heated cylinder using Schlieren imaging. This measurement allowed for the derivation of the experimental heat transfer coefficient, which was then compared with theoretical and CFD methods. Thus, the practicality of basic Schlieren imaging entails more than mere visualization - it also allows for distance and location identification in fluid flow. Another study conducted by Aung, Fletcher, and Sernas (Aung et al., 1972) investigated laminar natural convection between vertical flat plates with asymmetric heating conditions. Fringe patterns from Schlieren interferometer imaging, a closely related method to Schlieren imaging, yielded nearly identical local Nusselt numbers as numerical solutions. Aung, Fletcher, and Sernas' geometry is similar to the geometry studied in this paper.

For this study, the transition from the conduction regime to the convection regime has been studied. The system is characterized by the Rayleigh number $(R a)$.

$$
R a=\frac{g \beta \Delta T H^{3}}{\alpha \nu}
$$

$g$ is the acceleration due to gravity, $\beta$ is coefficient of thermal expansion of the air, $\Delta T$ is the difference between the highest and lowest temperatures on the cavity wall, $H$ is the height of the cavity, $\alpha$ is the thermal diffusivity of the air, and $\nu$ is the kinematic viscosity of the air.

Several parameters are used to classify the systems. The geometry of the cavity is represented by the aspect ratio $(A)$, where $A=H / W . H$ is the height of the cavity and $W$ is the width.

A number of studies have been done to characterize the enclosed rectangular geometry, most with different boundary conditions than this system. Previous modeling by Chenoweth and Paolucci (Chenoweth and Paolucci, 1986; Suslov and Paolucci, 1995) determined that the difference between steady motion and turbulence is dependent upon the aspect ratio of the cavity and the Rayleigh number of the heated air in the cavity. At an aspect ratio of 10 , as studied in this experiment, the onset of turbulence with uniform wall heating is expected to occur at a Rayleigh number of approximately $2.5 \cdot 10^{5}$ (Chenoweth and Paolucci, 1986). With the boundary conditions in this experiment Dillon et al. predicted the onset of steady oscillations at $2.5 \cdot 10^{7}$ and chaotic un-steady behavior at a Rayleigh number of approximately $5 \cdot 10^{7}$ (Dillon et al., 2013).

\section{METHODS}

\subsection{The Schlieren Setup}

The Schlieren imaging device used for this study was set up as a $\mathrm{z}$ type Schlieren. To create the Schlieren device, two 25.4-cm diameter parabolic mirrors with focal ratio $\mathrm{f} / 5$ were placed in parallel at approximately 1.5 meters apart. The mirrors were secured with custom-made wood housing. In addition to being in parallel, the mirrors were placed linearly so that light could be collimated through the test region. The table that secured this device was upgraded to hard plywood and steel from a less sturdy plywood and sheet metal design. This new design assured that no warps in the surface would distort the Schlieren image or misalign the mirrors.

At one focal length away from the first mirror, a black plastic screen with a pinhole was placed in front of a blue LED. This light was directed toward the mirror and positioned so that it was at a height that was level with the midpoints of the mirrors. At the other end of the light path, a razor blade was placed at one focal length from the other mirror. The Canon Powershot camera was positioned behind this blade so that half of the light would be filtered out via Foucault's knife-edge effect. Like the LED, the blade and camera were positioned to be level with the mirrors' midpoints as shown in Fig. 1.

\subsection{The Experimental Rectangular Cavity}

To examine the air cavity modeled by Dillon, et al. (Dillon et al., 2013). the experimental device was defined with opposing temperature profiles along the two vertical sides of the rectangle. The top and bottom sides were adiabatic (insulated), while the air in the cavity was enclosed. The final schematic of the experimental system is shown in Fig. 2 and the 


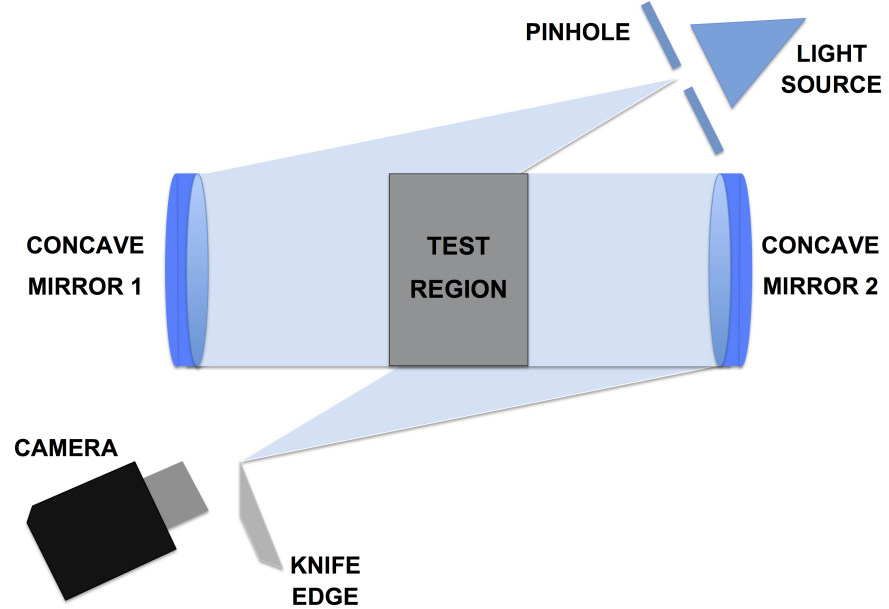

Fig. 1 Schematic of the z-type Schlieren setup adapted from (Kaessinger et al., 2014).

equipment is described in Table 2.

Because a Schlieren image of the 2-D airflow within the cavity was needed, the cavity could not be enclosed in the z-direction. To compensate, the experimental device was designed to be very long in the $\mathrm{z}$-direction relative to the $2-\mathrm{D}$ plane so that airflow in the inner region would be consistent and uniform. The cavity of the experimental system was then designed to be $56 \mathrm{~cm}$ deep and $20 \mathrm{~cm}$ tall. Two 2-mm-thick aluminum plates were used to form the vertical walls. These plates were mounted on a platform with L-brackets, where the L-brackets are on the exterior of the cavity, so they do not interact with the interior fluid motion. The walls were adjustable so that a variety of aspect ratios could be tested in the future. For this study, the walls were set $2 \mathrm{~cm}$ apart to yield an aspect ratio of 10. Panels of garolite with a gasket seal were set on the bottom and screwed to the top of the walls. The garolite made the top and bottom of the cavity roughly adiabatic.

Strip heaters were placed at the top outer side of one wall, and at the bottom of the other (named "Side A" and "Side B," respectively, for the purpose of this paper). The temperature of these heaters was regulated with PID controllers and thermocouples taped located beneath them. Additionally, thermocouples were taped fixed at 5,10 , and $15 \mathrm{~cm}$ from the bottom of each plate so that temperature profiles could be approximated. Another thin-wire thermocouple was threaded through the center of the air cavity to record temperature oscillations in the air.

There were some limitations with the experimental setup. For one, a large range of Rayleigh numbers could not be achieved due to conduction across the plates from the strip heaters. Also, temperature gradients across the two plates could not be made identical due to the effect of convective heat transfer. This meant that on Side B, where the heater was positioned at the bottom of the plate, both conduction and convection supplied heat to the upper portion of the plate. This effect yielded a smaller temperature gradient in comparison to Side A.

These limitations will be addressed in future iterations of this experiment, and additional tests will be conducted for a wider range of aspect ratios and boundary conditions. The current experimental setup is adaptable to a variety of aspect ratios, so this feature will be utilized in future experiments. A wider range of Rayleigh numbers can be reached with the addition of Peltier cooling chips. Incorporating these chips could also achieve more symmetrical temperature gradients across the opposing plates.
Table 2 List of equipment and materials used for the experimental Schlieren work.

\begin{tabular}{|l|}
\hline Description \\
\hline \hline GSO Parabolic Primary Mirror, \\
Dia. $25.4 \mathrm{~cm}$, Focal Ratio f/5 \\
\hline Blue UltraBright LED (rated for 3.0V at 20 mA) \\
\hline Canon Powershot ELPH $300 \mathrm{HS}$ \\
\hline 3.0 V DC power supply \\
\hline Variable voltage AC power regulator \\
\hline Omega High Temperature, \\
Dual-Element Heating Tape \\
\hline 1/16 DIN Micromega Autotune PID \\
Temperature/Process Controllers \\
\hline Single-edge razor blade \\
\hline 56x15-cm, 6-mm-thick sheet of garolite \\
\hline 56x20.3-cm, 2-mm-thick aluminum sheet \\
\hline
\end{tabular}

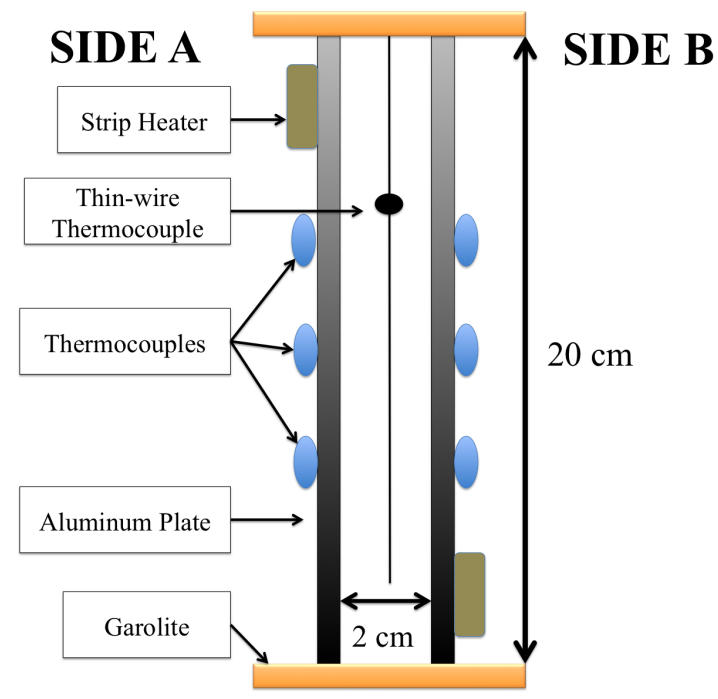

Fig. 2 Schematic of the cavity cross-section. Aluminum plates were 56 $\mathrm{cm}$ long in the direction perpendicular to this page.

\subsection{The Experimental Procedure}

The heaters were set to 35, 45, 55, and 65 degrees Celsius with the PID controllers. These temperatures corresponded to Rayleigh numbers of $0.41 \cdot 10^{7}, 0.66 \cdot 10^{7}, 0.87 \cdot 10^{7}$, and $1.03 \cdot 10^{7}$, respectively. Once heater temperatures reached steady state, the oscillating zone between the two cells was located using the Schlieren effect. The thin-wire thermocouple was adjusted so that the junction was the oscillating zone. Temperature data from this thermocouple was written to a measurement file via LabVIEW at 1/12-second intervals for 10 minutes. Simultaneously, data was recorded from the six outer thermocouples in 5-minute intervals. This process was repeated for all four heater temperatures. These six temperatures from the six external thermocouples were used to verify the desired boundary conditions, as shown in Fig. 3.

\section{EXPERIMENTAL RESULTS}

\subsection{Comparison to Prior CFD Modeling}

The Schlieren imaging device confirmed the existence of two convection cells and an oscillating zone within the cavity, as predicted by Dillon, et al. (Dillon et al., 2013). The oscillating cells were predicted to be symmetrical in the cavity as shown in Fig. 4.

In the present experimental work seen in Fig. 5, the oscillating zone 


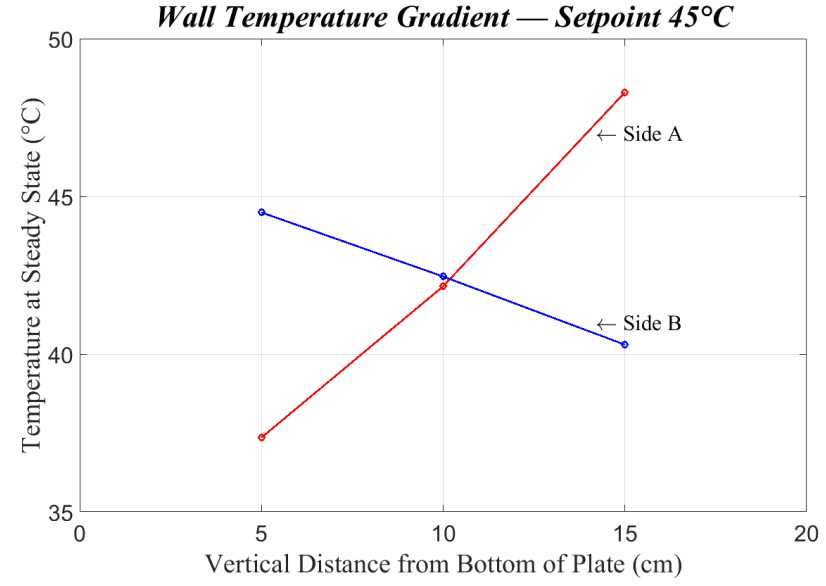

Fig. 3 Verification of the desired boundary conditions. As necessitated by the definition of this experiment, the heaters created a linear temperature across the two sides of the cavity.

was found to be about $6 \mathrm{~cm}$ from the top of the cavity. This location was roughly consistent for all four Rayleigh numbers tested. The location was higher in the cavity than in Dillon's CFD model (Dillon et al., 2013), which predicted two equal-sized cells with the oscillating zone in the center of the cavity. This discrepancy possibly indicates the need for adjustments to the existing CFD models to better reflect how this system behaves in reality. For example, the Boussinesq approximation may neglect imperfections in the real system. The slight imperfections in the experimental boundary conditions may also play a role in the higher oscillatory zone.

\subsection{Experimental Data}

As expected, the Rayleigh numbers corresponded to high heater set-point temperatures, because higher temperatures yielded greater temperature gradients. Mean inner temperature (as recorded at the oscillating zone) increased with the heater set-point, but not proportionately. This was most likely due to the lower average temperature in proportion to the higher heater temperatures. The average temperature at each Rayleigh number is shown in Table 3 and Fig. 6.

Table 3 Summary of the mean air temperatures and the standard deviations at the oscillating zone and their corresponding Rayleigh numbers.

\begin{tabular}{ccc}
\hline $\begin{array}{c}\text { Rayleigh } \\
\text { Number }\end{array}$ & $\begin{array}{c}\text { Oscillation Zone Mean } \\
\text { Temperature }\left[{ }^{\circ} \mathrm{C}\right]\end{array}$ & $\begin{array}{c}\text { Standard } \\
\text { Deviation }\left[{ }^{\circ} \mathrm{C}\right]\end{array}$ \\
\hline $0.41 \cdot 10^{7}$ & 35.91 & \pm 0.127 \\
$0.66 \cdot 10^{7}$ & 40.53 & \pm 0.689 \\
$0.87 \cdot 10^{7}$ & 47.23 & \pm 0.683 \\
$1.03 \cdot 10^{7}$ & 53.46 & \pm 0.850 \\
\hline
\end{tabular}

Oscillating zone temperature became visibly less steady as the Rayleigh number was increased. The standard deviation in the oscillating zone temperature temperature ranged from $\pm 0.127^{\circ} \mathrm{C}$ at $R a=0.41 \cdot 10^{7}$ to $\pm 0.850^{\circ} \mathrm{C}$ at $R a=1.03 \cdot 10^{7}$. Subsequently, changes in the temperature became larger and more rapid as the Rayleigh number increased, as shown in Fig. 6. There is a marked difference in the behavior of the low and high Rayleigh number experiments. At $R a=0.41 \cdot 10^{7}$ shows relatively constant temperature indicating no oscillations, while $R a=1.03 \cdot 10^{7}$ shows frequent, rapid swings in temperature. As expected, standard deviation of the temperatures generally increased with Rayleigh number, with the two intermediate Rayleigh numbers with comparable deviations.

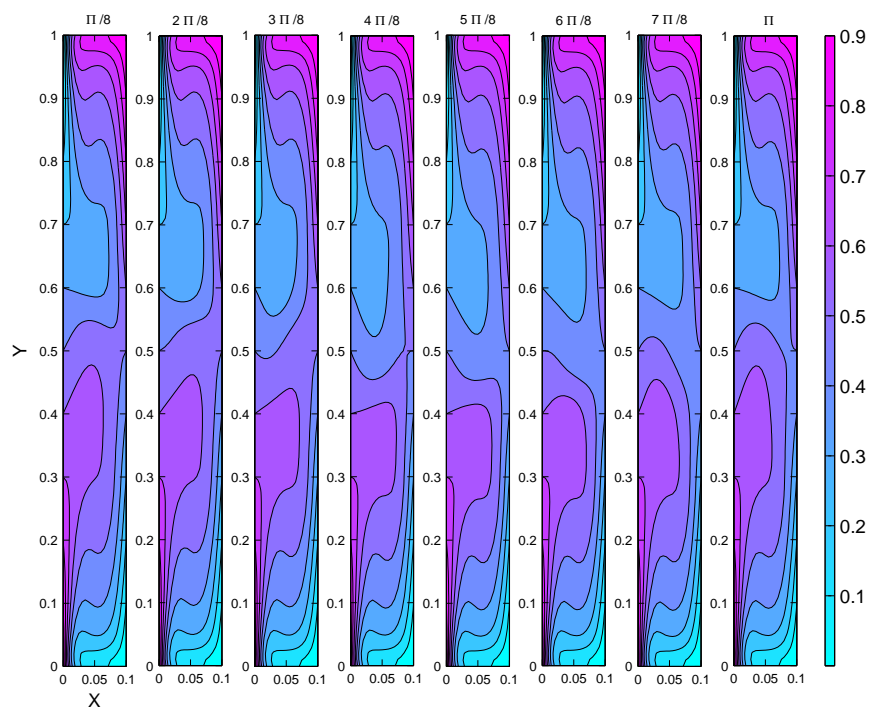

(a) $\Theta$

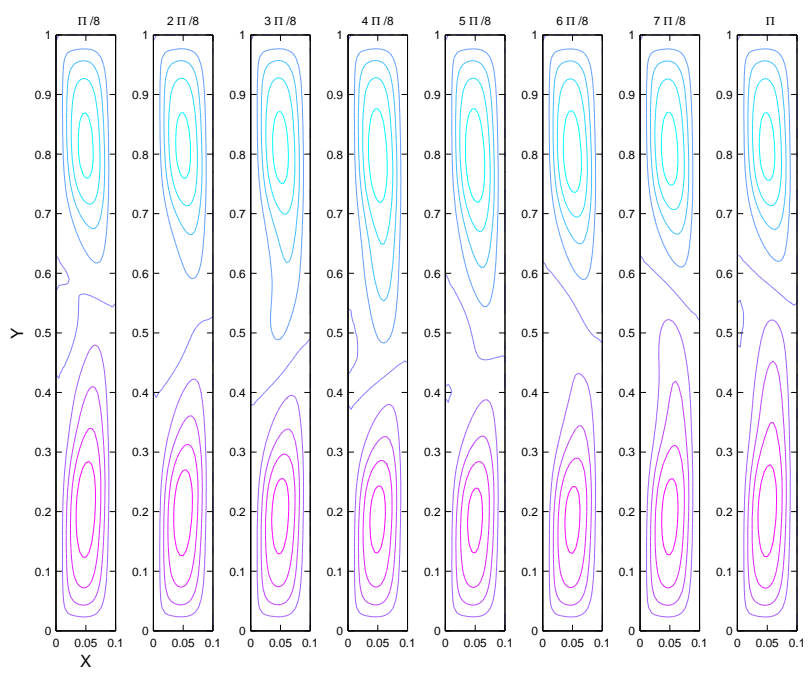

(b) $\psi$

Fig. 4 Sequential contour plot of the dimensionless temperature $\Theta$ (a) and stream function $\psi$ (b) for the rectangular cavity illustrating oscillation of the natural convection cells through one period (П). $R a=2.5 \times 10^{7}, A=10$. Adapted from Dillon et al. (Dillon et al., 2013).

To quantify the frequency behavior the spectral density function of the experimental data was calculated. The results are shown in Figure 7. For the lowest Rayleigh number the system is relatively steady and no frequencies with high power appear. As the Rayleigh number is increased frequencies with higher power occur, many showing harmonic behavior in the same range predicted by Dillon et al. (Dillon et al., 2013). However the behavior of the experimental system shows more complexity than the modeled system.

This data indicates that Schlieren imaging can be used to assist in characterizing phenomena in natural convection systems. Overall, the results from this experiment support CFD-based predictions by Dillon, et al., and transitional behavior predicted by Chenoweth and Paolucci for a system with different boundary conditions. 


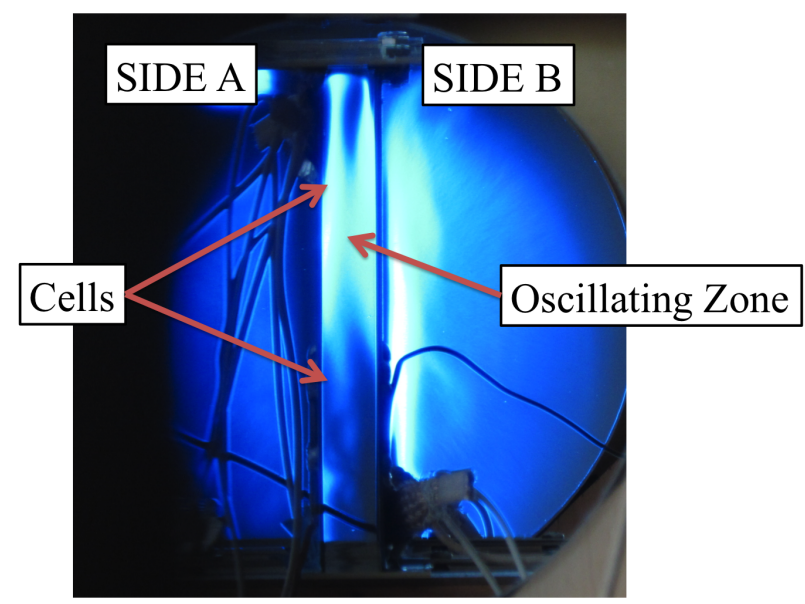

Fig. 5 Schlieren image of the heated cavity air density $(\rho)$. Note the two cells (darker) and oscillating zone (lighter) between the cells. Waste heat on the right side from the exposed strip heater is also shown, but occurs outside the cavity wall.

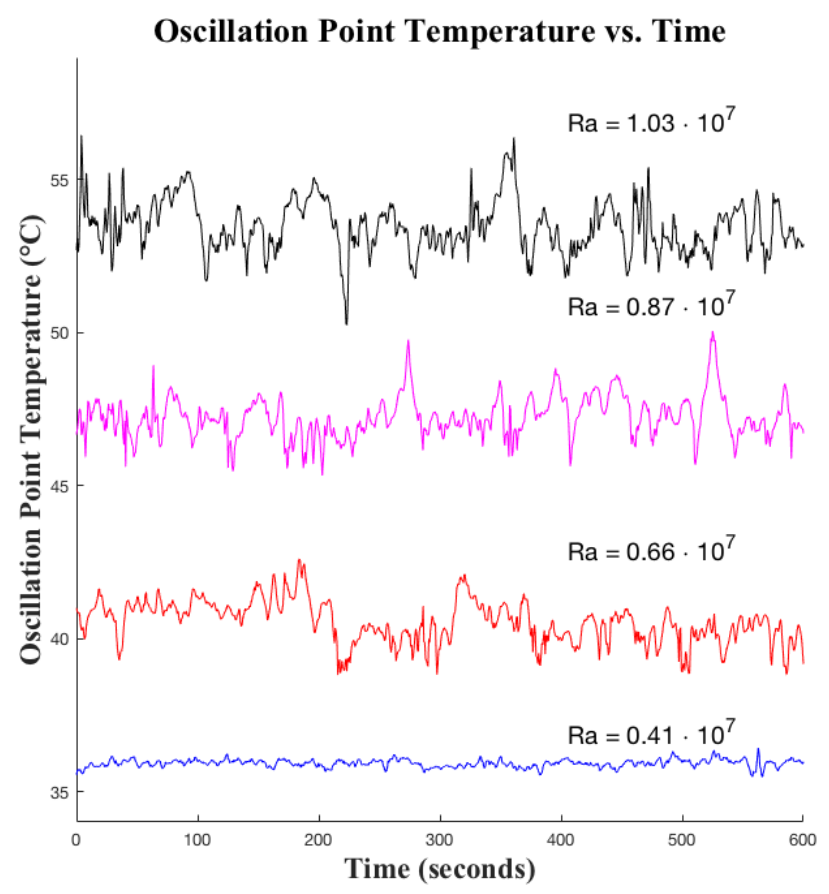

Fig. 6 Comparison of temperature oscillations at the oscillating zone for different Rayleigh numbers.

\section{CONCLUSIONS}

The results of this experiment have confirmed the existence of at least two convection cells in a rectangular cavity with non-isothermal boundary conditions. Furthermore, the results confirmed the oscillatory motion and interaction zone of these cells as predicted in CFD modeling by Dillon, et al. (Dillon et al., 2013). The interaction point between the two cells was higher in the air cavity than predicted by CFD modeling. This discrepancy could be due to a failure of the Boussinesq approximation to account for imperfections in the real system, or due to the limitations of the experimental test setup. Higher Rayleigh numbers indicated the
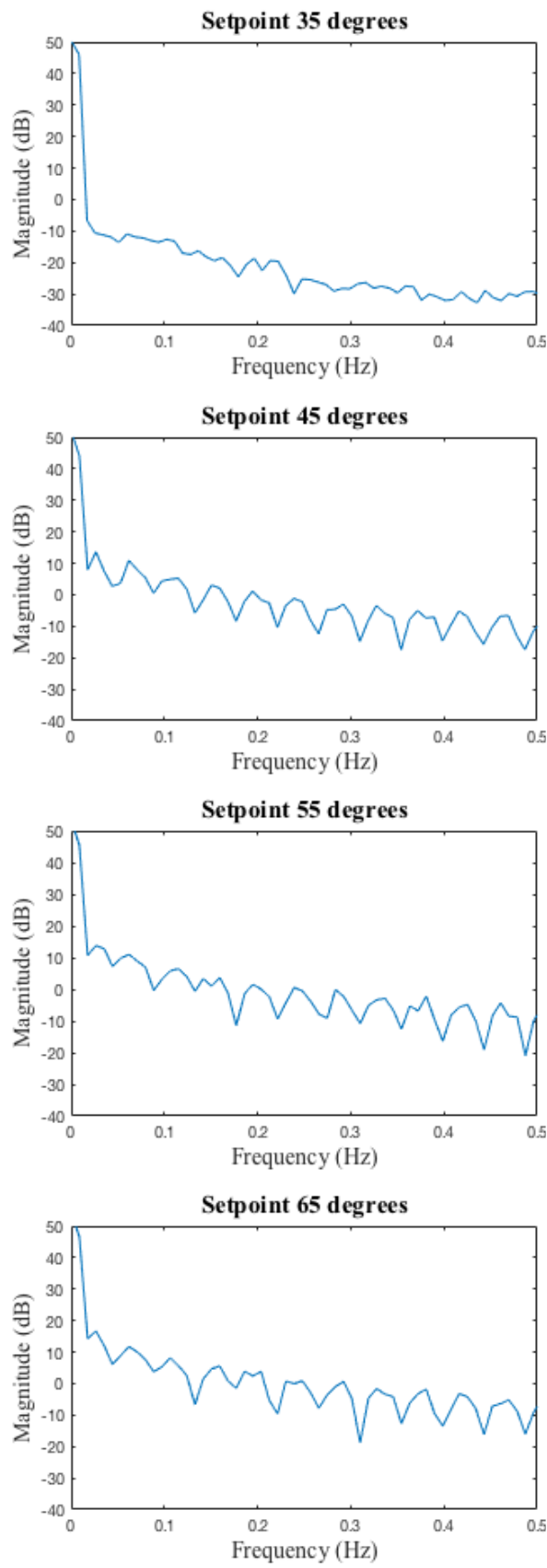

Fig. 7 Spectral density function for the experimental temperature data at each Rayleigh number.

beginnings of unsteady motion, confirming the system is transitioning to more complex behavior as expected. Ultimately, the results from this experiment demonstrated the ability of Schlieren imaging to help characterize the onset of turbulence in natural convection systems. In this case, Schlieren imaging successfully confirmed the existence of multiple oscillating air cells in a rectangular cavity with non-isothermal boundary conditions. Further characterizing turbulence in such systems could impact a variety of fields, including the improvement of air cooling in electronics and better understanding of air flow in buildings. 


\section{ACKNOWLEDGEMENTS}

Special thanks to Sharon Jones, Dean of the Shiley School of Engineering, for financially supporting this experiment, and to Jacob Amos and Jared Rees for assistance with the development of the experimental setup. Additional thanks to Hollis Crowder and Thomas Storey for lab support.

\section{NOMENCLATURE}

$g \quad$ acceleration of gravity

$H \quad$ height of the cavity

$h \quad$ heat transfer coefficient

$k \quad$ thermal conductivity

$\operatorname{Pr} \quad$ Prandlt number

$R a \quad$ Rayleigh number

$T$ temperature

$v \quad$ velocity

$W \quad$ width of the cavity

Greek Symbols

$\beta \quad$ coefficient of thermal expansion

$\delta_{t} \quad$ thermal boundary layer thickness

$\mu \quad$ dynamic viscosity

$\nu \quad$ kinematic viscosity

$\rho \quad$ density

$\psi \quad$ stream function

$\sigma \quad$ surface tension of liquid

$\Theta \quad$ dimensionless temperature $\left(\Theta=\frac{T-T_{c}}{\Delta T}\right)$

$t \quad$ time

\section{REFERENCES}

Aung, W., Fletcher, L., and Sernas, V., 1972, "Developing Laminar Free Convection between Vertical Flat Plates with Asymmetric Heating," International Journal of Heat and Mass Transfer, 15(11), 2293-2308, http://doi:10.1016/0017-9310(72)90048-8.

Chenoweth, D.R., and Paolucci, S., 1986, "Natural Convection in an Enclosed Vertical Air Layer with Large Horizontal Temperature Differences," Journal of Fluid Mechanics, 169(-1), 173, http://doi:10.1017/S0022112086000587.

Clark, R.P., and Toy, N., 1975, "Natural Convection Around the Human Head." The Journal of Physiology, 244(2), 283-93, http://doi:10.1113/jphysiol.1975.sp010797.

Dillon, H., Emery, A., and Mescher, A., 2013, "Analysis of Chaotic Natural Convection in a Tall Rectangular Cavity with Non-Isothermal Walls,"
Frontiers in Heat and Mass Transfer, 4(2), http://doi:10.5098/hmt.v4.2.3004.

Dillon, H.E., Emery, A.F., and Mescher, A.M., 2009, "Benchmark Comparison of Natural Convection in a Tall Cavity," COMSOL User Conference, 1-10, Boston, MA.

Kaessinger, J.C., Kors, K.C., Lum, J.S., Dillon, H.E., and Mayer, S.K., 2014, "Utilizing Schlieren Imaging to Visualize Heat Transfer Studies," American Society of Mechanical Engineers 2014 International Mechanical Engineering Conference, IMECE2014-38329, Montreal, Canada, http://doi:10.1115/IMECE2014-38329.

Lewis, H., Foster, A., Mullan, B., Cox, R., and Clark, R., 1969, "Aerodynamics of the Human Microenvironment," The Lancet, 293(7609), 12731277 , http://doi:10.1016/S0140-6736(69)92220-X.

Okhotsimskii, A., and Hozawa, M., 1998, "Schlieren Visualization of Natural Convection in Binary Gas-liquid Systems," Chemical Engineering Science, 53(14), 2547-2573, http://doi:10.1016/S0009-2509(98)00092-X.

Settles, G., 1985, "Colour-coding Schlieren Techniques for the Optical Study of Heat and Fluid Flow," International Journal of Heat and Fluid Flow, 6(1), 3-15, http://doi:10.1016/0142-727X(85)90024-4.

Settles, G., 2001, “Schlieren and Shadowgraph Techniques,” Tech. rep.

Suslov, S., and Paolucci, S., 1995, "Stability of Natural Convection Flow in a Tall Vertical Enclosure under Non-Boussinesq onditions," International Journal of Heat and Mass Transfer, 38(12), 2143-2157, http://doi:10.1016/0017-9310(94)00348-Y.

Tanda, G., Fossa, M., and Misale, M., 2014, "Heat Transfer Measurements in Water using a Schlieren Technique," International Journal of Heat and Mass Transfer, 71, 451-458, http://doi:10.1016/j.ijheatmasstransfer.2013.12.022.

Venkatakrishnan, L., and Meier, G., 2004, "Density Measurements using the Background Oriented Schlieren Technique," Experiments in Fluids, 37(2),

http://doi:10.1007/s00348-004-0807-1. 